\title{
A fiber-coupled quantum-dot on a photonic tip
}

Cadeddu, Davide; Teissier, Jean; Braakman, Floris R.; Gregersen, Niels; Stepanov, Petr; Gérard, Jean Michel; Claudon, Julien; Warburton, Richard J.; Poggio, Martino; Munsch, Mathieu

\section{Published in:}

Applied Physics Letters

Link to article, DOI:

$10.1063 / 1.4939264$

Publication date:

2016

Document Version

Publisher's PDF, also known as Version of record

Link back to DTU Orbit

Citation (APA):

Cadeddu, D., Teissier, J., Braakman, F. R., Gregersen, N., Stepanov, P., Gérard, J. M., Claudon, J., Warburton, R. J., Poggio, M., \& Munsch, M. (2016). A fiber-coupled quantum-dot on a photonic tip. Applied Physics Letters, 108, [011112]. https://doi.org/10.1063/1.4939264

\section{General rights}

Copyright and moral rights for the publications made accessible in the public portal are retained by the authors and/or other copyright owners and it is a condition of accessing publications that users recognise and abide by the legal requirements associated with these rights.

- Users may download and print one copy of any publication from the public portal for the purpose of private study or research.

- You may not further distribute the material or use it for any profit-making activity or commercial gain

- You may freely distribute the URL identifying the publication in the public portal 


\title{
A fiber-coupled quantum-dot on a photonic tip
}

\author{
Davide Cadeddu, ${ }^{1}$ Jean Teissier, ${ }^{1}$ Floris R. Braakman, ${ }^{1}$ Niels Gregersen, ${ }^{2}$ Petr Stepanov, ${ }^{3,4}$ \\ Jean-Michel Gérard, ${ }^{3,4}$ Julien Claudon, ${ }^{3,4}$ Richard J. Warburton, ${ }^{1}$ Martino Poggio, ${ }^{1}$ \\ and Mathieu Munsch ${ }^{1}$ \\ ${ }^{1}$ Department of Physics, University of Basel, Klingelbergstrasse 82, CH-4056 Basel, Switzerland \\ ${ }^{2}$ DTU Fotonik, Department of Photonics Engineering, Technical University of Denmark, Building 343, \\ DK-2800 Kongens Lyngby, Denmark \\ ${ }^{3}$ Université Grenoble Alpes, F-38100 Grenoble, France \\ ${ }^{4} C E A, I N A C-S P 2 M, 17$ rue des Martyrs, F-38054 Grenoble, France
}

(Received 28 October 2015; accepted 13 December 2015; published online 8 January 2016)

\begin{abstract}
We present the experimental realization of a quantum fiber-pigtail. The device consists of a semiconductor quantum-dot embedded into a conical photonic wire that is directly connected to the core of a fiber-pigtail. We demonstrate a photon collection efficiency at the output of the fiber of $5.8 \%$ and suggest realistic improvements for the implementation of a useful device in the context of quantum information. We also discuss potential applications in scanning probe microscopy. The approach is generic and transferable to other materials including diamond and silicon. (C) 2016 AIP Publishing LLC. [http://dx.doi.org/10.1063/1.4939264]
\end{abstract}

Semiconductor quantum-dots (QDs) are attractive single photon sources. They are robust, compact, and provide ondemand single photons at rates in the GHz range. ${ }^{1-3}$ Their potential in the context of quantum optics, however, relies on the fulfillment of several demanding criteria: ${ }^{4}$ high efficiency, high photon purity, and simple operation. Recent progress has nevertheless brought QDs close to such applications. Single-photon operation has been obtained in a compact, table-top Stirling machine, ${ }^{5}$ offering a low-cost and user-friendly solution. Thanks to the increasing quality of the epitaxial material, spectrally pure emission has been demonstrated. ${ }^{6}$ The last challenge that needs to be addressed is to efficiently couple the emitted light into a single mode fiber. Large progress in this direction has been made with the integration of QDs into micro and nano-scale photonic structures, such as cavities and waveguides, which allow the control of spontaneous emission. ${ }^{7-9}$ In the last few years, important efforts to position the QD in an optimal way ${ }^{10,11}$ and to minimize the diffraction of light at the output of photonic nanowires ${ }^{12}$ have pushed the collection efficiencies to values $\gtrsim 75 \%$ while maintaining a Gaussian spatial profile. ${ }^{13,14}$ These impressive results require, however, the use of objective lenses with large numerical apertures. In parallel, different strategies to couple the emitted light directly into a single mode fiber have emerged. ${ }^{15-18}$

We report here the direct coupling of QD single photons to an optical fiber. Our device, the quantum fiber-pigtail, consists of a QD embedded in a tapered photonic wire (PW), a photonic "trumpet," which is directly attached to the cleaved end of a single mode fiber. Thanks to adiabatic expansion of the guided mode confined in the PW, we achieve an external collection efficiency of $5.8 \%$ at the output of the fiber-pigtail. The result represents a proof-of-principle for an easy-to-operate single photon source, with potential applications in quantum optics or metrology. In this perspective, we discuss realistic improvements of the collection efficiency. Furthermore, easily addressable QDs at the end of a nanometer-scale tip have obvious potential as scanning probes. Possible applications include single photon near-field microscopy, ${ }^{19,20}$ deterministic quantum plasmonics, ${ }^{21}$ or electric field sensing. ${ }^{22}$ Finally, we emphasize that the approach is generic and can be transferred to other nanoscale emitters such as colored centers in diamond or silicon.

Our quantum emitter consists of a self-assembled InAs QD grown by molecular beam epitaxy. It is embedded $110 \mathrm{~nm}$ away from the sharp end of a $12 \mu \mathrm{m}$-long conical GaAs PW standing on a gold substrate, ${ }^{14}$ see Fig. 1(a). At the QD position, the small diameter enhances the coupling of the emitter to the guided modes while screening its coupling to lateral free-space modes. ${ }^{8}$ As the top facet is approached, the progressive increase in diameter allows for an adiabatic expansion of the guided modes and eventually leads to a better mode matching with the fiber. These conical wires are obtained through a top-down approach. Using e-beam lithography, we define a Ni hard mask consisting of arrays of disks with variable diameters. This is followed by a deep plasma etch conducted in a reactive ion-etching chamber. Finally, the remaining Ni mask is removed in a diluted nitric acid solution. $\mathrm{A} \mathrm{Si}_{3} \mathrm{~N}_{4}$ anti-reflection coating maximizes the transmission through the top facet. The resulting structures are shown in Figs. 1(a) and 1(b).

In order to realize a direct coupling between the photonic trumpet and a single mode fiber, we pick up an individual wire and glue it to the core of a standard fiber $\left(d_{\text {core }}=4.4 \mu \mathrm{m}\right.$, $n_{\text {core }}=1.4563$, and $n_{\text {cladding }}=1.4513$ ), as illustrated in Fig. 2(a). Initially, we fabricate micro-manipulators by tapering a glass needle down to a few micron thicknesses. The micro-manipulator is then used to pick up one wire at a time thanks to a combination of electrostatic and Van der Waals forces (see Fig. 1(b)). Observations with a scanning electron microscope (SEM) indicate that the cleaving point lies at the interface between the PW and the substrate, within an estimated error of $\pm 10 \mathrm{~nm}$ (the instrument's resolution). With a second micromanipulator, we re-orient the PW into the appropriate direction (top facet facing downwards), Fig. 1(c). We 

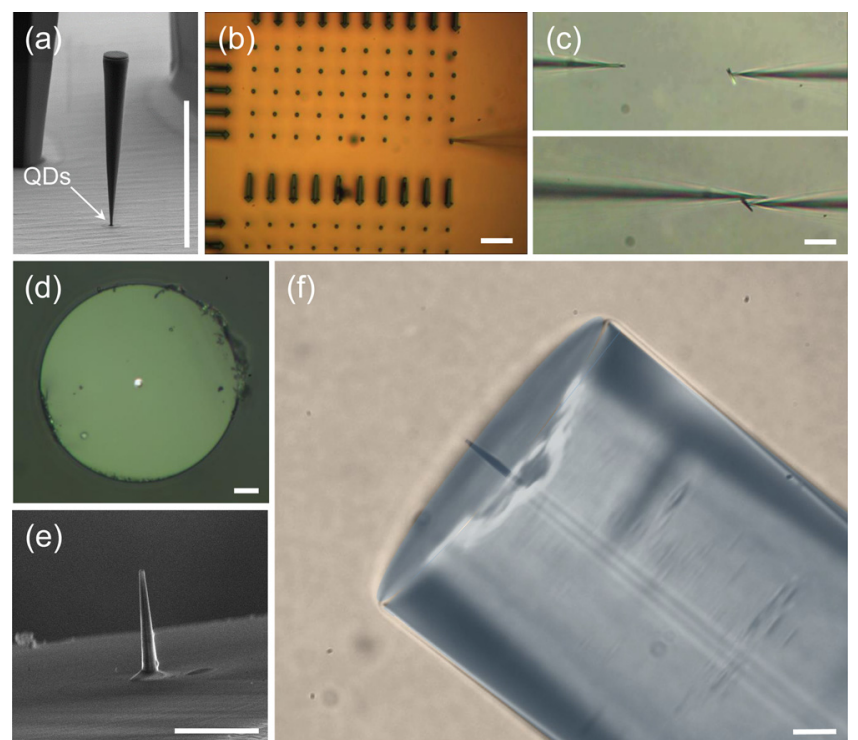

FIG. 1. Fabrication procedure. (a) SEM picture of the photonic trumpet after etching and removal of the Ni mask. (b) Removing a single PW from its original substrate with glass micro-manipulator (right side). (c) Orientation of the PW for subsequent gluing onto the fiber. (d) Top view of the bare fiber with a drop of UV glue (bright spot) deposited at its center. (e) SEM picture of the fiber-wire connection. The drop of glue can be seen at the base of the PW. (f) Side view of the final device (optical microscope image). The white scale bars represent $10 \mu \mathrm{m}$.

then approach the cleaved facet of a fiber-pigtail and deposit a drop of low fluorescence UV glue onto its core, Fig. 1(d). Finally, we bring the wire and the fiber into contact with an alignment precision on the order of $1 \mu \mathrm{m}$ and illuminate with UV light to harden the glue. The resulting structure is shown in Figs. 1(e) and 1(f). ${ }^{23}$ It is robust and resistant to cycling to cryogenic temperatures, ${ }^{24}$ two important points for future applications.

The device is tested at cryogenic temperature by plunging it directly into liquid He. The QDs are excited non-resonantly with a $\mathrm{CW}$ laser diode and the photoluminescence (PL) is analyzed with a spectrometer and a high efficiency Si-based CCD camera $\left(\eta_{\text {det }}=27 \%\right.$ at $\lambda=950 \mathrm{~nm}$ ), see Fig. 2(a). A typical spectrum is shown in Fig. 2(b) for an excitation in the bulk ( $\left.\lambda_{\text {laser }}=780 \mathrm{~nm}\right)$. We identify a peak associated to the GaAs nanowire and a series of sharp lines corresponding to several QDs. Importantly, we observe significant heating as we increase the non-resonant power. This is evidenced as a quadratic shift of the QD energies in Fig. 2(c) and indicates a poor heat dissipation in the device, despite the surrounding liquid He. A simple way to avoid this problem is to create electron-hole pairs directly in the InGaAs wetting layer connecting the QDs $\left(\lambda_{\text {laser }}=830 \mathrm{~nm}\right)$. In this case, we minimize the amount of absorbed light and observe no heating effect over the range of useful excitation powers (see Fig. 2(c)). This second scenario was used for all the results presented below.

We focus on a PW featuring a diameter of $440 \mathrm{~nm}$ at the QD's position and $1.8 \mu \mathrm{m}$ at the top facet. The wire sustains the guided modes associated with the first six orders and contains approximately 50 QDs distributed over a spectral bandwidth of about $50 \mathrm{~nm}$. In the low energy tail, we identify, in
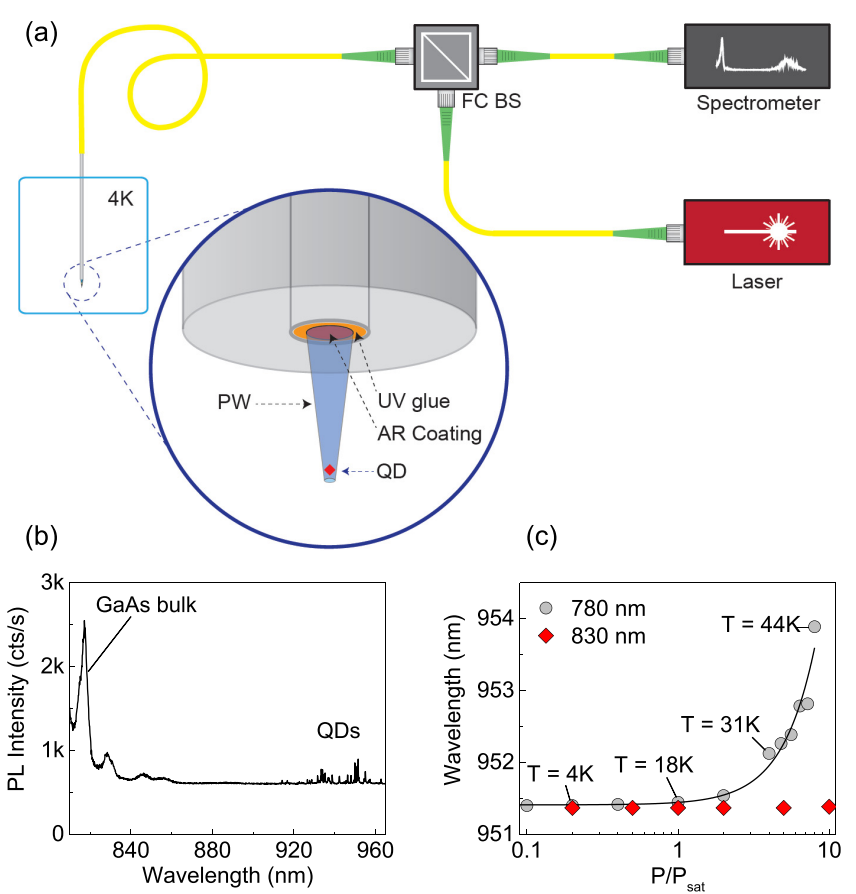

FIG. 2. General properties. (a) The quantum fiber-pigtail is cooled down to $4 \mathrm{~K}$ in liquid He. The QDs are excited with a CW laser diode $(\lambda=830 \mathrm{~nm}$ or $780 \mathrm{~nm})$. The photoluminescence (PL) is analyzed with a spectrometer and a CCD camera (not shown). (b) Typical PL spectrum for excitation in the GaAs bulk. (c) Power-induced heating of the PW for excitation in the GaAs bulk (gray circles). Excitation in the wetting layer (red diamonds) results in negligible heating. The abscissa has been normalized to the saturation power $P_{\text {sat }}$. The solid line is a quadratic fit to the data.

particular, a bright complex labeled QD1, which consists of an excitonic transition $\mathrm{X}$ (possibly a charged exciton) and a red-shifted biexciton XX at high power (see Fig. 3(a)).

To confirm the quantum nature of the emitted signal, we perform an auto-correlation measurement on the exciton using a standard Hanbury-Brown Twiss experiment with a set of two avalanche photo-diodes. The result, shown in Fig. 3(b), reveals a pronounced dip at zero delay, characteristic of anti-bunched emission. The data are very well reproduced by the auto-correlation function of a perfect 2-level emitter with a lifetime of $1 / \gamma_{X}=1.2$ ns convoluted with the detector's response (Gaussian with full-width-half-maximum of $\sim 400 \mathrm{ps}$ ).
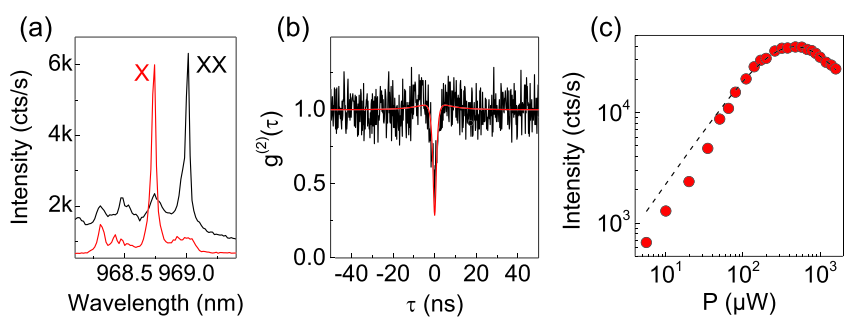

FIG. 3. Characterization of a single QD. (a) QD1 spectra at low $(P=125 \mu \mathrm{W}$, in red) and high $(P=1.2 \mathrm{~mW}$, in black) powers. $\mathrm{X}$ and $\mathrm{XX}$ correspond to the exciton and the biexciton. (b) Auto-correlation signal of $\mathrm{X}$ $(P=64 \mu \mathrm{W})$. The data are normalized to the average coincidence counts per time bin. The dip at zero delay is the signature of a single quantum emitter. The red solid line is a fit to the 2-level atom result including the detector time-jitter ( 400 ps). (c) Power dependence of the exciton PL intensity. The dashed line is a fit using Eq. (1). 
To evaluate the extraction efficiency $\epsilon$ of our quantum fiber-pigtail, we record the PL intensity as we increase the excitation power. As we saturate the $\mathrm{X}$ transition, we observe a maximum count rate of $\sim 40 \mathrm{kcts} / \mathrm{s}$, Fig. 3(c). The data are fitted using a simple three-level model that takes the biexciton into account. Denoting $\gamma_{\mathrm{X}}$ and $\gamma_{\mathrm{XX}}$ the decay rates for the exciton and the biexciton, the detected PL intensity is given by ${ }^{25}$

$$
I_{\mathrm{X}, \operatorname{det}}(P)=\frac{I_{\mathrm{sat}}}{1+\frac{\alpha P}{\gamma_{\mathrm{X}}}+\frac{\gamma_{\mathrm{XX}}}{\alpha P}},
$$

with $I_{\text {sat }}$ being the intensity at saturation and $\alpha$ being a coefficient which translates the measured excitation power into an effective pumping rate. Using $\gamma_{X}=0.84 \mathrm{GHz}$ from the auto-correlation measurement and $\gamma_{\mathrm{XX}} \sim 2 \gamma_{\mathrm{X}}$, we obtain a very good agreement with our experimental data for $I_{\text {sat }}$ $=149 \mathrm{kcts} / \mathrm{s}$ and $\alpha=2.5 \times 10^{-3} \mathrm{GHz} / \mu \mathrm{W}$. The relationship between the detected flux and the emission rate simply reads

$$
I_{\text {sat }}=\epsilon \eta_{t} \eta_{\text {det }} \gamma_{\mathrm{X}}
$$

where $\eta_{t}$ corresponds to the overall transmission between the fiber-pigtail and the detector. Using a reference tunable laser diode set at $970 \mathrm{~nm}$, we find $\eta_{t}=6 \% \pm 3.5 \%$, which yields a collection efficiency $\epsilon=5.8 \% \pm 3.3 \%$. The given value includes all losses, for instance, the finite coupling of the QD to the waveguide-modes propagating in the upward direction, the imperfect wire-to-fiber mode matching, and the transmission losses. This result can still be improved, but we stress that it is already more than one order of magnitude superior to the value one would obtain from QDs in the bulk. It therefore constitutes a proof-of-principle for our approach of integrating a quantum light source to a standard optical fiber.

We estimate the quality of the fabrication by evaluating the expected maximum efficiency at the output of the fiber-pigtail. Using Lumerical FDTD Solutions, we calculate the fraction of power radiated through the top facet of the PW by an embedded dipole point-source. The result is multiplied by the overlap between the computed profile of the electromagnetic field (at the top facet) and the mode of the fiber. For a PW with the above-mentioned dimensions and a QD on axis, we find $\epsilon=9.2\left(\begin{array}{c}+0.4 \\ -0.7\end{array}\right) \%$, where the error bar comes from the $\pm 10 \mathrm{~nm}$ uncertainty on the distance between the QD and the cleaved facet. Our experimental result is in agreement with the theoretical prediction. This calculation shows that we have met the main challenges, namely, positioning the QD close to the axis and aligning the center of the PW with the core of the fiber.

We believe that the proposed device has the potential to relax some of the constraints associated with the use of single QDs in quantum optics experiment. Integrating it into a compact closed cycle cryostat would result in a quantum light source taking the form of a "black-box," with single photons directly available at the output of a standard single mode fiber. However, in this context, a useful device should exhibit a larger efficiency.

To optimize $\epsilon$, a natural strategy is to operate the tapered PW in the single mode regime. This choice simultaneously ensures high emission probabilities into the fundamental guided mode $\left(\mathrm{HE}_{11}\right)$ and optimum mode matching between the PW and the fiber. In this regime, a lower bound to the coupling efficiency is given by $\epsilon=\beta_{u p} \mathcal{T}_{\mathrm{HE}_{11}} \mathcal{T}_{\mathrm{f}}$. Here, $\beta_{u p}$ is the fraction of photons emitted in the guided mode propagating towards the fiber; $\mathcal{T}_{\mathrm{HE}_{11}}$ is the taper modal transmission; and $\mathcal{I}_{\mathrm{f}}$ is the taper facet to fiber facet modal transmission. Interestingly, a simple dielectric facet with a diameter of $0.25 \lambda$ provides an amplitude reflectivity of $60 \% .{ }^{26}$ By placing the QD at an anti-node of the resulting interference pattern, $\beta_{\text {up }}$ can be increased up to $75 \%$ for an on-axis emitter. Moreover, a taper with larger height $h$ and top facet $d_{t}$ leads to a spectacular improvement of the coupling efficiency. As an example, the device reported in Ref. $27(h=27 \mu \mathrm{m}$ and $d_{t}=5 \mu \mathrm{m}$ ) features $\mathcal{T}_{\mathrm{HE}_{11}} \mathcal{T}_{\mathrm{f}}=59 \%$. Thus, combining this geometry and an appropriately located bottom facet (e.g., using a sacrificial layer), $\epsilon=44 \%$ is already within reach, without major technological developments. Regarding further improvements, $\beta_{u p}>90 \%$ is achievable with an additional post metallic mirror, ${ }^{26}$ and $\mathcal{T}_{\mathrm{f}}$ can be further improved with larger taper dimensions. A taper with $h=80 \mu \mathrm{m}$ and $d_{t}=7 \mu \mathrm{m}$ would then bring $\epsilon$ above $70 \%$. As a final comment, we note that one may also tailor the properties of the fiber and release some constraints on the PW geometry.

Our device also presents attractive features as a surface scanning probe. We point out two examples of possible applications along this line. The first one concerns quantum plasmonics. Surface plasmon polaritons (SPPs) represent a possible way of building integrated quantum optics circuits at the nanoscale. ${ }^{28}$ Our device could be used to transfer quantum information from the QD to propagative SPPs simply by bringing the sharp tip of our photonic wire into close proximity with a metallic nanostructure $(d<\lambda / 2)$, Fig. 4(a). Compared to the

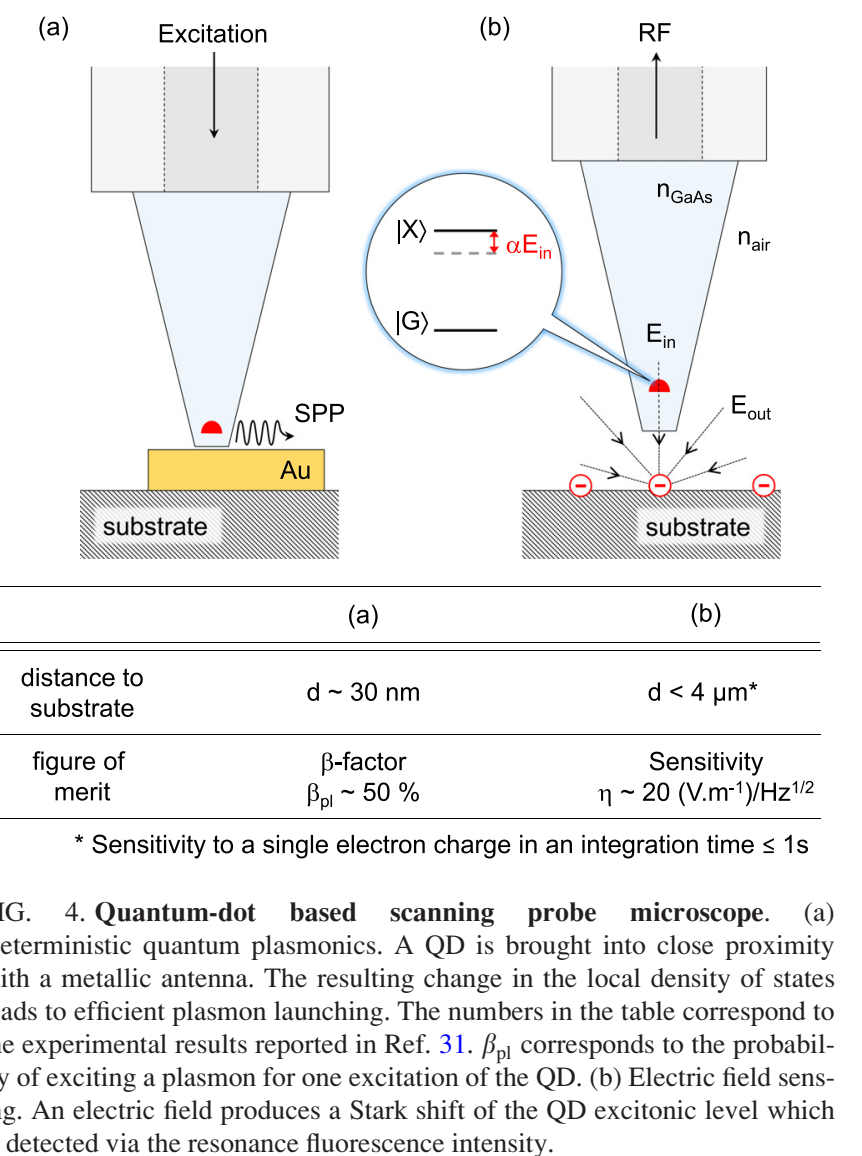


previous work, ${ }^{29,30}$ this solution presents more flexibility and would allow scanning of the sample surface or bringing the quantum emitter to a specific location, while controlling its exact distance to a given metallic antenna. The possibility of positioning our probe at will presents a significant advantage to explore the effect of the near-field environment on the emission properties of a QD. In particular, recent experiments have shown that control over the distance (as well as over the QD orientation) could lead to launching plasmons with probabilities approaching $50 \% .^{31}$ The present device is thus likely to find applications in the field of quantum plasmonics, in particular, when more complex plasmonic circuits come to the fore. $^{32,33}$

A second application concerns using the QD at the apex of the wire as an ultra-sensitive electric field sensor, Fig. 4(b). The idea is that an electric field $E_{\text {in }}$ results in a Stark shift that can be detected through a change in intensity of QD resonance fluorescence. The noise spectrum of the QD resonance fluorescence signal reveals the noise spectrum of the local electric field. ${ }^{6}$ In the linear regime and for a vertical electric field, the Stark shift is given by $\Delta \nu=\alpha^{-1} E_{\text {in }} / h$, where $\alpha \sim 0.3 \mu \mathrm{eV} /(\mathrm{kV} / \mathrm{m})^{22,34}$ and $h$ is the Planck constant. Assuming a detection floor limited by shot noise, we derive the sensitivity of the QD sensor

$$
\eta=\frac{\alpha^{-1}}{\partial \dot{n}(\nu) / \partial \Delta \nu} \sqrt{2 \dot{n}(\nu)}
$$

where $\dot{n}(\nu)=\frac{\dot{n}_{0}}{1+(2 \nu / \Gamma)^{2}}$ is the resonance fluorescence count rate. Assuming a count rate at resonance $\dot{n}_{0}=1 \mathrm{MHz}$, a linewidth of $\Gamma=5 \mu \mathrm{eV}$, and taking into account the reduction in electric field amplitude due to its penetration into a high refractive index material $\left(n_{\mathrm{GaAs}}=3.5\right)$

$$
E_{\text {in }} \propto \frac{1}{n^{2}} E_{\text {out }},
$$

we obtain sensitivities to an external electric field $E_{\text {out }}$ as high as $\eta \sim 20(\mathrm{~V} / \mathrm{m}) / \sqrt{\mathrm{Hz}}$. This corresponds to detecting a single charge at a distance of $4 \mu \mathrm{m}$ within a $1 \mathrm{~s}$ integration time, or alternatively to detecting a single charge at a distance of $150 \mathrm{~nm}$ in just $1 \mathrm{~ms}$. Importantly, we point out the very large sensor bandwidth, which is ultimately limited by the spontaneous emission rate and can thus approach the $\mathrm{GHz}$ range in the case of standard InGaAs QDs.

QD electric field sensors display sensitivities which compete with those of a single-electron transistor, while minimally affecting the system being detected. ${ }^{22}$ The scanning capability offered by our quantum pigtail makes it an appealing alternative to existing devices. We finally note that the quantum pigtail could be straightforwardly attached to a tuning fork force sensor ${ }^{35}$ facilitating atomic force microscopy with the tapered photonic wire as tip, and that the translation of this concept to NV centers in diamond would enable the creation of a scanning magnetic field sensor. ${ }^{36,37}$

In conclusion, we present a device concept for an alignment-free, easy-to-operate source of single photons. A semiconductor photonic trumpet containing quantum dots is attached to the facet of a single-mode optical fiber. As a figure of merit, we have evaluated the extraction efficiency at the output of the fiber-pigtail (5.8\%) and demonstrated good agreement with numerical calculations. We have shown that realistic adjustment could lead to values $>70 \%$. Importantly, the fabricated device is robust and operates well in a cryogenic environment. Finally, we have underlined its potential in the context of deterministic quantum plasmonics and electric field sensing.

This work was supported by an ERC Grant (NWscan, Grant No. 334767), the Swiss Nanoscience Institute (Project P1207), and the NCCR QSIT. J.C. and J.-M.G. acknowledge the support of the European Union Seventh Framework Program 209 (FP7/2007-2013) under Grant Agreement No. 601126210 (HANAS) and the European Metrology Research Program (EMRP) [project SIQUTE (Contract EXL02)]. N.G. acknowledges the support from the Danish Research Council for Technology and Production (Project LOQIT, Grant No. 4005-00370B). The photonic trumpet fabrication was carried out in the "Plateforme technologique amont" and CEA LETI MINATEC/DOPT clean rooms.

We thank Patrick Maletinsky and Nicolas Sangouard for discussions, and Andrea Mehlin for technical assistance.

${ }^{1}$ P. Michler, A. Kiraz, C. Becher, W. V. Schoenfeld, P. M. Petroff, L. D. Zhang, E. Hu, and A. Imamoglu, Science 290, 2282 (2000), ISSN 00368075 .

${ }^{2}$ C. Santori, M. Pelton, G. Solomon, Y. Dale, and Y. Yamamoto, Phys. Rev. Lett. 86, 1502 (2001).

${ }^{3}$ E. Moreau, I. Robert, J. M. Grard, I. Abram, L. Manin, and V. ThierryMieg, Appl. Phys. Lett. 79, 2865 (2001).

${ }^{4}$ N. Sangouard and H. Zbinden, J. Mod. Opt. 59, 1458 (2012).

${ }^{5}$ A. Schlehahn, L. Krüger, M. Gschrey, J.-H. Schulze, S. Rodt, A. Strittmatter, T. Heindel, and S. Reitzenstein, Rev. Sci. Instrum. 86, 013113 (2015).

${ }^{6}$ A. V. Kuhlmann, J. Houel, A. Ludwig, L. Greuter, D. Reuter, A. D. Wieck, M. Poggio, and R. J. Warburton, Nat. Phys. 9, 570 (2013), ISSN 1745-2473.

${ }^{7}$ J. M. Gérard, B. Sermage, B. Gayral, B. Legrand, E. Costard, and V. Thierry-Mieg, Phys. Rev. Lett. 81, 1110 (1998).

${ }^{8}$ J. Bleuse, J. Claudon, M. Creasey, N. S. Malik, J.-M. Gérard, I. Maksymov, J.-P. Hugonin, and P. Lalanne, Phys. Rev. Lett. 106, 103601 (2011).

${ }^{9}$ T. Lund-Hansen, S. Stobbe, B. Julsgaard, H. Thyrrestrup, T. Sünner, M. Kamp, A. Forchel, and P. Lodahl, Phys. Rev. Lett. 101, 113903 (2008).

${ }^{10}$ A. Dousse, L. Lanco, J. Suffczyński, E. Semenova, A. Miard, A. Lemaître, I. Sagnes, C. Roblin, J. Bloch, and P. Senellart, Phys. Rev. Lett. 101, 267404 (2008).

${ }^{11}$ M. E. Reimer, G. Bulgarini, N. Akopian, M. Hocevar, M. B. Bavinck, M. A. Verheijen, E. P. A. M. Bakkers, L. P. Kouwenhoven, and V. Zwiller, Nat. Commun. 3, 737 (2012).

${ }^{12}$ J. Claudon, N. Gregersen, P. Lalanne, and J.-M. Gérard, ChemPhysChem 14, 2353 (2013), ISSN 1439-7641.

${ }^{13}$ O. Gazzano, S. Michaelis de Vasconcellos, C. Arnold, A. Nowak, E. Galopin, I. Sagnes, L. Lanco, A. Lemaitre, and P. Senellart, Nat. Commun. 4, 1425 (2013).

${ }^{14}$ M. Munsch, N. S. Malik, E. Dupuy, A. Delga, J. Bleuse, J.-M. Gérard, J. Claudon, N. Gregersen, and J. Mørk, Phys. Rev. Lett. 110, 177402 (2013).

${ }^{15}$ X. Xu, I. Toft, R. T. Phillips, J. Mar, K. Hammura, and D. A. Williams, Appl. Phys. Lett. 90, 061103 (2007).

${ }^{16}$ F. Haupt, S. S. R. Oemrawsingh, S. M. Thon, H. Kim, D. Kleckner, D. Ding, D. J. Suntrup, P. M. Petroff, and D. Bouwmeester, Appl. Phys. Lett. 97, 131113 (2010).

${ }^{17}$ T. Schröder, A. W. Schell, G. Kewes, T. Aichele, and O. Benson, Nano Lett. 11, 198 (2011).

${ }^{18}$ T. G. Tiecke, K. P. Nayak, J. D. Thompson, T. Peyronel, N. P. de Leon, V. Vuletić, and M. D. Lukin, Optica 2, 70 (2015). 
${ }^{19}$ J. Michaelis, C. Hettich, J. Mlynek, and V. Sandoghdar, Nature 405, 325 (2000).

${ }^{20}$ A. Cuche, A. Drezet, Y. Sonnefraud, O. Faklaris, F. Treussart, J.-F. Roch, and S. Huant, Opt. Express 17, 19969 (2009).

${ }^{21}$ A. Cuche, O. Mollet, A. Drezet, and S. Huant, Nano Lett. 10, 4566 (2010).

${ }^{22}$ A. N. Vamivakas, Y. Zhao, S. Fält, A. Badolato, J. M. Taylor, and M. Atatüre, Phys. Rev. Lett. 107, 166802 (2011).

${ }^{23}$ See supplementary material at http://dx.doi.org/10.1063/1.4939264 for a video illustrating the fabrication process.

${ }^{24}$ We performed up to 3 cycles with the same device without loss of signal.

${ }^{25}$ M. Munsch, A. Mosset, A. Auffèves, S. Seidelin, J. P. Poizat, J.-M. Gérard, A. Lemaître, I. Sagnes, and P. Senellart, Phys. Rev. B 80, 115312 (2009).

${ }^{26}$ I. Friedler, C. Sauvan, J. P. Hugonin, P. Lalanne, J. Claudon, and J. M. Gérard, Opt. Express 17, 2095 (2009).

${ }^{27}$ P. Stepanov, A. Delga, N. Gregersen, E. Peinke, M. Munsch, J. Teissier, J. Mørk, M. Richard, J. Bleuse, J.-M. Gérard et al., Appl. Phys. Lett. 107, 141106 (2015).
${ }^{28}$ M. S. Tame, K. R. McEnery, S. K. Ozdemir, J. Lee, S. A. Maier, and M. S. Kim, Nat. Phys. 9, 329 (2013).

${ }^{29}$ A. V. Akimov, A. Mukherjee, C. L. Yu, D. E. Chang, A. S. Zibrov, P. R. Hemmer, H. Park, and M. D. Lukin, Nature 450, 402 (2007).

${ }^{30}$ G. Bracher, K. Schraml, M. Blauth, J. Wierzbowski, N. C. López, M. Bichler, K. Müller, J. J. Finley, and M. Kaniber, J. Appl. Phys. 116, 033101 (2014).

${ }^{31}$ M. L. Andersen, S. Stobbe, A. S. Sorensen, and P. Lodahl, Nat. Phys. 7, 215 (2011).

${ }^{32}$ Y. Fang, Z. Li, Y. Huang, S. Zhang, P. Nordlander, N. J. Halas, and H. Xu, Nano Lett. 10, 1950 (2010).

${ }^{33}$ H. Wei, Z. Wang, X. Tian, M. Kall, and H. Xu, Nat. Commun. 2, 387 (2011).

${ }^{34}$ J. Houel, A. V. Kuhlmann, L. Greuter, F. Xue, M. Poggio, B. D. Gerardot, P. A. Dalgarno, A. Badolato, P. M. Petroff, A. Ludwig et al., Phys. Rev. Lett. 108, 107401 (2012).

${ }^{35}$ K. Karrai and R. Grober, Appl. Phys. Lett. 66, 1842 (1995).

${ }^{36}$ C. L. Degen, Appl. Phys. Lett. 92, 243111 (2008).

${ }^{37}$ G. Balasubramanian, I. Y. Chan, R. Kolesov, M. Al-Hmoud, J. Tisler, C. Shin, C. Kim, A. Wojcik, P. R. Hemmer, A. Krueger et al., Nature 455, 648 (2008). 